\title{
Time optimal quantum control of two-qubit systems
}

\author{
LI Bin ${ }^{1,2}$, YU ZuHuan ${ }^{1}$, FEI ShaoMing ${ }^{1,3}$ and LI-JOST XianQing ${ }^{3}$ \\ ${ }^{1}$ School of Mathematical Sciences, Capital Normal University, Beijing 100048, China; \\ ${ }^{2}$ School of Mathematics and Statistics, Northeast Normal University, Changchun 130024, China; \\ ${ }^{3}$ Max-Planck-Institute for Mathematics in the Sciences, Leipzig 04103, Germany
}

\begin{abstract}
We study the optimal quantum control of heteronuclear two-qubit systems described by a Hamiltonian containing both nonlocal internal drift and local control terms. We derive an explicit formula to compute the minimum time required to steer the system from an initial state to a specified final state. As applications the minimal time to implement Controlled-NOT gate, SWAP gate and Controlled-U gate is calculated in detail. The experimental realizations of these quantum gates are explicitly presented.
\end{abstract}

Key wards: Time optimal quantum control, heteronuclear two-spin system, local invariants

PACS: 03.67.-a, 32.80.Qk

\section{Introduction}

The optimal control of quantum systems [1, 2, 3] plays important roles in quantum computation and quantum information processing [4]. For instance, the nuclear magnetic resonance (NMR) used in information processing relies on a limited set of control variables in order to create desired unitary transformations that manipulate an ensemble of nuclear spins to transfer coherence between coupled spins in multidimensional NMR-experiments [5], or to implement quantum-logic gates in NMR quantum computers [6]. There have been many rigorous results on the optimal control of spin systems from numerical calculations, together with some experimental realizations in NMR systems [7, 8, 9, 10, 11].

Nevertheless, it has been still a challenging problem to determine the minimum time analytically for the implementation of an arbitrary given unitary transformation. Based on Cartan 
decomposition of unitary operators, the authors in ref. [12] studied the minimum time required to steer the system from some initial sate to a specified final state for a given controllable right invariant system, described by a Hamiltonian containing both a nonlocal internal or drift term, and a local control term. An elegant analytical characterization of such time optimal control in spin systems has been presented. However, since the Cartan decomposition of a unitary operator is not unique, the formula given in ref. [12] can not be operationally applied to compute the minimal time for a detailed given unitary operator.

In this paper, by using the local invariants associated with the local equivalent transformation of unitary operators [13, 14], we give an operational approach to compute the minimal time required to implement a given unitary operator for the heteronuclear system [15]. For examples, we explicitly compute the minimal time for several important quantum gates such as controlled-NOT, SWAP and controlled-U ones. Moreover, based on the optimal Cartan decomposition of these unitary operators in the derivation of the minimal time, we get the corresponding ways to to realize these quantum gates experimentally, with the control Hamiltonian explicitly given.

The state of a quantum system is described by a density matrix $\rho$. The state $\rho(0)$ at time zero evolves into the state $\rho(t)$ at time $t, \rho(t)=U(t) \rho(0) U^{\dagger}(t)$ for some unitary operator $U(t)$. The unitary operator $U(t)$ is determined by the Hamiltonian of the system $H(t)$ satisfying the time-dependent Schrödinger equation,

$$
\dot{U}(t)=-i H(t) U(t)
$$

with $U(0)=I$ the identity operator. For finite-dimensional quantum systems, $H(t)$ is a Hermitian matrix of the form,

$$
H(t)=H_{d}+\sum_{i=1}^{m} v_{i}(t) H_{i},
$$

where $H_{d}$ is called the drift Hamiltonian which is internal to the system, and $\sum_{i=1}^{m} v_{i}(t) H_{i}$ is the control Hamiltonian such that the coefficients $v_{i}(t)$ can be externally manipulated [12].

The key problem in optimal time control of a quantum system is to find the minimal time $t^{*}$ required for the system to reach the final state $\rho\left(t^{*}\right)$ from a initial state $\rho(0)$, namely, to implement a unitary operator $U\left(t^{*}\right)$.

The problem can be investigated according to the algebraic properties related to the unitary group actions. Let $G$ be a Lie group and $g$ its corresponding Lie algebra. Let $K$ denote a compact 
closed subgroup of $G$, and $l$ the Lie algebra of right invariant vector fields on $K$. There is an one-to-one correspondence between the vector fields $T_{e}(G)$ and the tangent spaces $T_{e}(K)$, denoted by $g$ and $l$ respectively, $g=l \oplus p, p=l^{\perp}$. For a real semi-simple Lie algebra $g$, one has a Cartan decomposition, $[l, l] \subset l,[p, l]=p,[p, p] \subset l$. If $s$ is a subalgebra of $g$ contained in $p$, then $s$ is Abelian as $[p, p] \subset l$. A maximal Abelian subalgebra contained in $p$ is called a Cartan subalgebra. The homogeneous coset space $G / K$ is a differential manifold. The Lie group $G$ has similarly a Cartan decomposition, $G=K e^{s} K$.

\section{Heteronuclear two-spin system}

We consider the typical and most interesting optimal time control problem of a heteronuclear two-spin (two-qubit) system [15], with the Hamiltonian (21) given by

$$
\begin{aligned}
& H_{d}=\frac{\pi}{2} J \sigma_{z}^{1} \sigma_{z}^{2}, \\
& H_{1}=\pi \sigma_{x}^{1}, \quad H_{2}=\pi \sigma_{y}^{1}, \quad H_{3}=\pi \sigma_{x}^{2}, \quad H_{4}=\pi \sigma_{y}^{2},
\end{aligned}
$$

where $\sigma_{x}^{\alpha}, \sigma_{y}^{\alpha}$ and $\sigma_{z}^{\alpha}$ are Pauli matrices acting on the $\alpha$ th quibt, $\alpha=1,2$ and $J$ is the coupling constant of the system.

In this case the problem is related to the special unitary group $G=U(4)$. As an arbitrary two-qubit gate can be decomposed as the product of a gate $U_{1} \in S U(4)$ and a global phase shift $e^{i \theta}$, $\theta \in \mathbb{R}$, the problem is reduced to the study of the group $S U(4)$ in stead of the group $U(4)$. The Lie algebra $s u(4)$ of $S U(4)$ has a Cartan decomposition $g=p \oplus l$ with $l=\operatorname{span} \frac{i}{2}\left\{\sigma_{x}^{1}, \sigma_{y}^{1}, \sigma_{z}^{1}, \sigma_{x}^{2}, \sigma_{y}^{2}, \sigma_{z}^{2}\right\}$ and

$$
p=\operatorname{span} \frac{i}{2}\left\{\sigma_{x}^{1} \sigma_{x}^{2}, \sigma_{x}^{1} \sigma_{y}^{2}, \sigma_{x}^{1} \sigma_{z}^{2}, \sigma_{y}^{1} \sigma_{x}^{2}, \sigma_{y}^{1} \sigma_{y}^{2}, \sigma_{y}^{1} \sigma_{z}^{2}, \sigma_{z}^{1} \sigma_{x}^{2}, \sigma_{z}^{1} \sigma_{y}^{2}, \sigma_{z}^{1} \sigma_{z}^{2}\right\}
$$

together with the Cantan subalgebra, $s=\operatorname{span} \frac{i}{2}\left\{\sigma_{x}^{1} \sigma_{x}^{2}, \sigma_{y}^{1} \sigma_{y}^{2}, \sigma_{z}^{1} \sigma_{z}^{2}\right\}$.

Since the set of all the local gates $K$ is a connected Lie group $S U(2) \otimes S U(2)$ in $S U(4)$, $l=\operatorname{span} \frac{i}{2}\left\{\sigma_{x}^{1}, \sigma_{y}^{1}, \sigma_{z}^{1}, \sigma_{x}^{2}, \sigma_{y}^{2}, \sigma_{z}^{2}\right\}$ is just the Lie subalgebra corresponding to $K$. Therefore $U \in S U(4)$ can be decomposed as:

$$
U=k_{1} \exp \left\{\frac{i}{2}\left(c_{1} \sigma_{x}^{1} \sigma_{x}^{2}+c_{2} \sigma_{y}^{1} \sigma_{y}^{2}+c_{3} \sigma_{z}^{1} \sigma_{z}^{2}\right)\right\} k_{2}
$$

where $k_{1}, k_{2} \in S U(2) \otimes S U(2)$, and $c_{1}, c_{2}, c_{3} \in \mathbb{R}$. 
When the control terms in the Hamiltonian are large enough compared with the internal couplings, any single-qubit operation can be achieved almost instantaneously. It has been proved in [12] that for the Hamiltonian system described by eq. (3), the minimal time to implement a quantum gate $U$ of the form (44) is given by

$$
t^{*}=\frac{1}{\pi J} \min \sum_{i=1}^{3} c_{i}, \quad c_{i}>0 .
$$

Since for given $U$, its decompositions of the form (4) are not unique, it is a challenging problem to find the minimum of $\sum_{i=1}^{3} c_{i}$.

To find an analytical formula of $t^{*}$, we consider the local invariants and local equivalent classes in $U(4)$. Two unitary transformations $U, U_{1} \in U(4)$ are said to be locally equivalent if they satisfy, $U=k_{1} U_{1} k_{2}$, for some $k_{1}, k_{2} \in U(2) \otimes U(2)$, which defines a set of invariants under such equivalent transformations. These invariants can be expressed as [14],

$$
G_{1}=\frac{\operatorname{tr}^{2}[m(U)]}{16 \operatorname{det} U}, \quad G_{2}=\frac{\operatorname{tr}^{2}[m(U)]-\operatorname{tr}\left[m^{2}(U)\right]}{4 \operatorname{det} U},
$$

where $m(U)=U_{B}^{T} U_{B}, U_{B}=O^{\dagger} U O$, and

$$
O=\frac{1}{\sqrt{2}}\left(\begin{array}{cccc}
1 & 0 & 0 & i \\
0 & i & 1 & 0 \\
0 & i & -1 & 0 \\
1 & 0 & 0 & -i
\end{array}\right)
$$

As $U$ can be expressed in the form (44), one has the invariants [14],

$$
G_{1}=a+i b, \quad G_{2}=c
$$

where

$$
\begin{aligned}
& a=\cos ^{2} c_{1} \cos ^{2} c_{2} \cos ^{2} c_{3}-\sin ^{2} c_{1} \sin ^{2} c_{2} \sin ^{2} c_{3}, \\
& b=\frac{1}{4} \sin 2 c_{1} \sin 2 c_{2} \sin 2 c_{3}, \\
& c=4 \cos ^{2} c_{1} \cos ^{2} c_{2} \cos ^{2} c_{3}-4 \sin ^{2} c_{1} \sin ^{2} c_{2} \sin ^{2} c_{3}-\cos 2 c_{1} \cos 2 c_{2} \cos 2 c_{3} .
\end{aligned}
$$

Our main idea is to find the solution $c_{1}, c_{2}$ and $c_{3}$ from $G_{1}=a+i b, G_{2}=b$, according to the local invariants $a, b$ and $c$, so that the value $\sum_{i=1}^{3} c_{i}$ will be independent of the detailed Cartan expression (4). 
It is direct to verify that $\sqrt{a^{2}+b^{2}}=\cos ^{2} c_{1} \cos ^{2} c_{2} \cos ^{2} c_{3}+\sin ^{2} c_{1} \sin ^{2} c_{2} \sin ^{2} c_{3}$. Therefore we have

$$
\begin{aligned}
l \cos ^{2} c_{1} \cos ^{2} c_{2} \cos ^{2} c_{3} & =\frac{1}{2}\left(\sqrt{a^{2}+b^{2}}+a\right), \\
\sin ^{2} c_{1} \sin ^{2} c_{2} \sin ^{2} c_{3} & =\frac{1}{2}\left(\sqrt{a^{2}+b^{2}}-a\right) .
\end{aligned}
$$

However, by using the formula $\cos ^{2} \alpha+\sin ^{2} \alpha=1$, from (10) we also have

$$
\begin{aligned}
\cos ^{2} c_{1} \cos ^{2} c_{2} \cos ^{2} c_{3}= & 1-\left(\sin ^{2} c_{1}+\sin ^{2} c_{2}+\sin ^{2} c_{3}\right)-\sin ^{2} c_{1} \sin ^{2} c_{2} \sin ^{2} c_{3} \\
& +\left(\sin ^{2} c_{1} \sin ^{2} c_{2}+\sin ^{2} c_{1} \sin ^{2} c_{3}+\sin ^{2} c_{2} \sin ^{2} c_{3}\right) .
\end{aligned}
$$

Hence one gets

$$
\left(\sin ^{2} c_{1}+\sin ^{2} c_{2}+\sin ^{2} c_{3}\right)-\left(\sin ^{2} c_{1} \sin ^{2} c_{2}+\sin ^{2} c_{1} \sin ^{2} c_{3}+\sin ^{2} c_{2} \sin ^{2} c_{3}\right)=1-\sqrt{a^{2}+b^{2}} .
$$

Moreover, eq. (11) can be written as $\cos 2 c_{1} \cos 2 c_{2} \cos 2 c_{3}=4 a-c$. While

$$
\begin{aligned}
\cos 2 c_{1} \cos 2 c_{2} \cos 2 c_{3}= & \left(1-2 \sin ^{2} c_{1}\right)\left(1-2 \sin ^{2} c_{2}\right)\left(1-2 \sin ^{2} c_{3}\right) \\
= & 1-2\left(\sin ^{2} c_{1}+\sin ^{2} c_{2}+\sin ^{2} c_{3}\right)-8 \sin ^{2} c_{1} \sin ^{2} c_{2} \sin ^{2} c_{3} \\
& +4\left(\sin ^{2} c_{1} \sin ^{2} c_{2}+\sin ^{2} c_{1} \sin ^{2} c_{3}+\sin ^{2} c_{2} \sin ^{2} c_{3}\right) .
\end{aligned}
$$

Therefore we obtain

$$
\left(\sin ^{2} c_{1}+\sin ^{2} c_{2}+\sin ^{2} c_{3}\right)-2\left(\sin ^{2} c_{1} \sin ^{2} c_{2}+\sin ^{2} c_{1} \sin ^{2} c_{3}+\sin ^{2} c_{2} \sin ^{2} c_{3}\right)=\frac{1+c}{2}-2 \sqrt{a^{2}+b^{2}} .
$$

From eqs. (12) and (13), we have

$$
\left\{\begin{array}{l}
\sin ^{2} c_{1}+\sin ^{2} c_{2}+\sin ^{2} c_{3}=1+\frac{1-c}{2} \\
\sin ^{2} c_{1} \sin ^{2} c_{2}+\sin ^{2} c_{1} \sin ^{2} c_{3}+\sin ^{2} c_{2} \sin ^{2} c_{3}=\sqrt{a^{2}+b^{2}}+\frac{1-c}{2} .
\end{array}\right.
$$

From eqs. (14) and (11), we see that $\sin ^{2} c_{1}, \sin ^{2} c_{2}$ and $\sin ^{2} c_{3}$ can be considered as the solutions of the following cubic equation,

$$
x^{3}+p x^{2}+q x+r=\left(x-\sin ^{2} c_{1}\right)\left(x-\sin ^{2} c_{2}\right)\left(x-\sin ^{2} c_{3}\right)=0,
$$

where

$$
p=-\left(1+\frac{1-c}{2}\right), \quad q=\sqrt{a^{2}+b^{2}}+\frac{1-c}{2}, \quad r=-\frac{1}{2}\left(\sqrt{a^{2}+b^{2}}-a\right) .
$$

Set $X=x+p / 3$. The cubic equation becomes

$$
X^{3}+P X+Q=0
$$


where

$$
\left\{\begin{array}{l}
P=q-\frac{p^{2}}{3}=-\frac{1}{12}\left(c^{2}+3\right)+\sqrt{a^{2}+b^{2}} \\
Q=\frac{2 p^{3}}{27}-\frac{p q}{3}+r=\frac{1}{108}\left(c^{3}-9 c\right)+\frac{a}{2}-\frac{c}{6} \sqrt{a^{2}+b^{2}} .
\end{array}\right.
$$

To deal with eq. (16), we consider two elementary functions $Y=X^{3}$ and $Y=-P X-Q$ in $\mathbb{R}^{2}$. These two curves may intersect at one, two or three points with respect to different values of $P$ and $Q$. First, for the most special case: half the curve $Y=X^{3}$ tangents to the line $Y=-P X-Q$, one has one single real solution $X_{1}$ and another two real ones $X_{2}=X_{3}$,

$$
\begin{aligned}
X^{3}+P X+Q & =\left(X-X_{1}\right)\left(X-X_{2}\right)^{2} \\
& =X^{3}-\left(X_{1}+2 X_{2}\right) X^{2}+\left(2 X_{1} X_{2}+X_{2}^{2}\right) X-X_{1} X_{2}^{2}=0
\end{aligned}
$$

and also $X_{1}+2 X_{2}=0,2 X_{1} X_{2}+X_{2}^{2}=P,-X_{1} X_{2}^{2}=Q$. When $P$ and $Q$ satisfy the condition $P^{3} / 27+Q^{2} / 4=0$, the solutions of eq. (16) are

$$
X_{1}=-2(Q / 2)^{1 / 3}, \quad X_{2}=X_{3}=(Q / 2)^{1 / 3}
$$

Second, eq. (16) has three different real solutions when the inequality $P^{3} / 27+Q^{2} / 4<0$ is satisfied,

$$
X_{1}=-\frac{2 \sqrt{-3 P}}{3} \cos \frac{\theta}{3} ; \quad X_{2}, X_{3}=-\frac{2 \sqrt{-3 P}}{3} \cos \frac{\theta}{3} \pm \frac{\sqrt{3}}{3} \sin \frac{\theta}{3}
$$

where $\theta=\arccos T$ and $T=27 Q /\left(2(-3 P)^{3 / 2}\right) \in(-1,1)$.

According to the Shengjin's formulas, eq. (16) may have one single real solution and two imaginary solutions when the inequality $P^{3} / 27+Q^{2} / 4>0$ holds. Since in our case $P^{3} / 27+Q^{2} / 4 \leq$ 0 is always satisfied, there will be no imaginary solutions.

Combining the above results, we have

[Theorem] For the system (21) with the two-qubit Hamiltonian given by eq. (3), the minimal time to implement a unitary operator $U$ is given by

$$
t^{*}(U)=\frac{1}{\pi J} \min \sum_{i=1}^{3} c_{i}=\frac{1}{\pi J} \sum_{i=1}^{3} \arcsin \sqrt{X_{i}+\frac{3-c}{6}},
$$

where

$$
X_{1}=-2\left(\frac{Q}{2}\right)^{\frac{1}{3}}, \quad X_{2}=X_{3}=\left(\frac{Q}{2}\right)^{\frac{1}{3}}
$$

if $P^{3} / 27+Q^{2} / 4=0$; and

$$
X_{1}=-\frac{2 \sqrt{-3 P}}{3} \cos \frac{\theta}{3} ; \quad X_{2}, X_{3}=-\frac{2 \sqrt{-3 P}}{3} \cos \frac{\theta}{3} \pm \frac{\sqrt{3}}{3} \sin \frac{\theta}{3},
$$


if $P^{3} / 27+Q^{2} / 4<0$.

\section{Applications}

We have presented an analytical formula to compute the minimal time required to implement an arbitrary given unitary operation for two-qubit system (3). Two-qubit operations are the most fundamental ones in quantum computation and quantum information processing. As examples, here we compute the minimal time for several important two-qubit gates.

Example 1. Controlled-NOT gate $\quad C_{N O T}=|0\rangle\left\langle 0\left|\otimes I_{2}+\right| 1\right\rangle\langle 1| \otimes(|1\rangle\langle 0|+| 0\rangle\langle 1|)$, where $I_{2}$ is the $2 \times 2$ identity matrix. From eq. (5) we obtain $G_{1}=0, G_{2}=1$. That is, $a=b=0, c=1$ due to eq. (6). Hence from eq. (17) we have $P=-1 / 3, Q=-2 / 27$. We have $P^{3} / 27+Q^{2} / 4=0$, $X_{1}=2 / 3, X_{2}=X_{3}=-1 / 3$, and $c_{1}=\pi / 2, c_{2}=c_{3}=0$. Therefore the minimal time is given by $t^{*}\left(C_{N O T}\right)=\frac{1}{\pi J} \sum_{i=1}^{3} c_{i}=\frac{1}{2 J}$.

To optimally implement the gate $C_{n o t}$ experimentally, one has to find the Cartan decomposition of $C_{n o t}$ which fulfils $t^{*}\left(C_{N O T}\right)=\frac{1}{2 J}$. Let us assume

$$
\exp \left(\frac{\pi i}{4}\right) C n o t=k_{1} \exp \left(\frac{\pi i}{4} \sigma_{x} \otimes \sigma_{x}\right) k_{2}
$$

for some $k_{1}, k_{2} \in S U(2) \otimes S U(2)$. Note that $\exp \left(\frac{\pi i}{4} \sigma_{x} \otimes \sigma_{x}\right)=\frac{\sqrt{2}}{2}\left(I_{2}+i \sigma_{x} \otimes \sigma_{x}\right)$. The problem is to compute $k_{1}$ and $k_{2}$ in the following equation,

$$
(1+i)\left(\begin{array}{cc}
I_{2} & 0 \\
0 & \sigma_{x}
\end{array}\right)=k_{1}\left(\begin{array}{cc}
I_{2} & i \sigma_{x} \\
i \sigma_{x} & I_{2}
\end{array}\right) k_{2} .
$$

Set $k_{1}=A \otimes B, k_{2}=C \otimes D$, with $A=\left(a_{i j}\right), C=\left(c_{i j}\right), B, D \in S U(2)$. A direct computation yields

$$
k_{1}\left(\begin{array}{cc}
I_{2} & i \sigma_{x} \\
i \sigma_{x} & I_{2}
\end{array}\right) k_{2}=\left(\begin{array}{ll}
f_{11} & f_{12} \\
f_{21} & f_{22}
\end{array}\right),
$$

where

$$
\begin{aligned}
& f_{11}=\left(a_{11} c_{11}+a_{12} c_{21}\right) B D+i\left(a_{12} c_{11}+a_{11} c_{21}\right) B \sigma_{x} D, \\
& f_{12}=\left(a_{11} c_{12}+a_{12} c_{22}\right) B D+i\left(a_{12} c_{12}+a_{11} c_{22}\right) B \sigma_{x} D, \\
& f_{21}=\left(a_{21} c_{11}+a_{22} c_{21}\right) B D+i\left(a_{22} c_{11}+a_{21} c_{21}\right) B \sigma_{x} D, \\
& f_{22}=\left(a_{21} c_{12}+a_{22} c_{22}\right) B D+i\left(a_{22} c_{12}+a_{21} c_{22}\right) B \sigma_{x} D .
\end{aligned}
$$

From eq. (19) one has $f_{12}=f_{21}=0$, namely

$$
\begin{aligned}
& \left(a_{11} c_{12}+a_{12} c_{22}\right) I_{2}+i\left(a_{12} c_{12}+a_{11} c_{22}\right) \sigma_{x}=0 \\
& \left(a_{21} c_{11}+a_{22} c_{21}\right) I_{2}+i\left(a_{22} c_{11}+a_{21} c_{21}\right) \sigma_{x}=0 .
\end{aligned}
$$


By detailed analysis one obtains

$$
C=\frac{\sqrt{2}}{2}\left(\begin{array}{cc}
\exp (i \theta) & -\exp (-i \theta) \\
\exp (i \theta) & \exp (-i \theta)
\end{array}\right) \quad \text { and } A=\frac{\sqrt{2}}{2}\left(\begin{array}{cc}
\exp (i \beta) & \exp (i \beta) \\
-\exp (-i \beta) & \exp (-i \beta)
\end{array}\right) .
$$

From the expressions of $f_{11}$ and $f_{22}$ we have further

$$
D=\left(\begin{array}{cc}
0 & 1 \\
-1 & 0
\end{array}\right) \text { and } B=\frac{\sqrt{2}}{2}\left(I_{2}+i \sigma_{x}\right)\left(\begin{array}{cc}
0 & 1 \\
-1 & 0
\end{array}\right) \text {. }
$$

Therefore the Cartan decomposition of $C_{\text {not }}$ reads,

$$
\begin{aligned}
\text { Cnot }= & \exp \left(-\frac{\pi i}{4}\right)\left(\exp \left(-\frac{\pi i}{4} \sigma_{y}\right) \exp \left(\frac{\pi i}{4} \sigma_{x}\right) \otimes \exp \left(\frac{\pi i}{4} \sigma_{x}\right) \exp \left(\frac{\pi i}{2} \sigma_{y}\right)\right) . \\
& \exp \left(\frac{\pi i}{4} \sigma_{x} \otimes \sigma_{x}\right)\left(\exp \left(\frac{\pi i}{4} \sigma_{y}\right) \otimes \exp \left(-\frac{\pi i}{2} \sigma_{y}\right)\right) .
\end{aligned}
$$

To find the detailed way to implement $C_{n o t}$ experimentally, we expand the factor $\exp \left(\frac{\pi i}{4} \sigma_{x} \otimes \sigma_{x}\right)$ by using the following formula,

$$
\exp \left(\frac{i}{4} \sigma_{x} \otimes \sigma_{x}\right)=\left(\exp \left(-\frac{\pi i}{4} \sigma_{y}\right) \otimes \exp \left(\frac{\pi i}{4} \sigma_{y}\right)\right) \exp \left(-\frac{\pi i}{4} \sigma_{z} \otimes \sigma_{z}\right)\left(\exp \left(\frac{\pi i}{4} \sigma_{y}\right) \otimes \exp \left(\frac{-\pi i}{4} \sigma_{y}\right)\right) .
$$

Denote $T_{m}=\sigma_{m} \otimes 1$ and $S_{m}=1 \otimes \sigma_{m}, m=x, y, z$. We can rewrite the Cartan decomposition of $C_{n o t}$ as,

$C_{\text {not }}=\exp \left(\frac{-\pi}{4} i\right) \exp \left(-\frac{\pi}{4} i T_{y}\right) \exp \left(\frac{\pi}{4} i\left(T_{x}+S_{x}\right)\right) \exp \left(-\frac{\pi}{4} i\left(T_{y}+S_{y}\right)\right) \exp \left(-\frac{\pi}{4} i T_{z} S_{z}\right) \exp \left(\frac{\pi}{4} i\left(2 T_{y}+S_{y}\right)\right)$.

From the Schödinger equation (11) and the Hamiltonian (2), (3), we see that the unitary operator $C_{n o t}$ can be implemented, up to a global phase, by manipulating the control Hamiltonian such that

$$
H(t)=\left\{\begin{array}{lll}
H_{d}-\frac{N}{2}\left(H_{2}+\frac{H_{4}}{2}\right), & t \in\left[0, \frac{1}{N}\right] ; \\
H_{d}, & t \in\left[\frac{1}{N}, \frac{1}{N}+\frac{1}{2 J}\right] ; \\
H_{d}+\frac{N}{4}\left(H_{2}+H_{4}\right), & t \in\left[\frac{1}{N}+\frac{1}{2 J}, \frac{2}{N}+\frac{1}{2 J}\right] ; \\
H_{d}-\frac{N}{4}\left(H_{1}+H_{3}\right), & t \in\left[\frac{2}{N}+\frac{1}{2 J}, \frac{3}{N}+\frac{1}{2 J}\right] ; \\
H_{d}+\frac{N}{4} H_{2}, & t \in\left[\frac{3}{N}+\frac{1}{2 J}, \frac{4}{N}+\frac{1}{2 J}\right],
\end{array}\right.
$$

where $N$ is a real parameter.

The parameter $N$ in the control Hamiltonian should be large enough, $N \rightarrow \infty$, so that the drift Hamiltonian $H_{d}$ can be ignored during all the local unitary evolutions, and the time needed 
for local unitary evolutions can be put to zero. The finite time needed to implement $C_{n o t}$ is in the second step at time interval $t \in\left[\frac{1}{N}, \frac{1}{N}+\frac{1}{2 J}\right]$. For $N \rightarrow \infty$, one reaches the optimal time $\frac{1}{2 J}$.

Example 2. SWAP gate For the gate Swap,

$$
S w a p=\frac{1}{2}\left(\begin{array}{cc}
I_{2}+\sigma_{z} & \sigma_{x}+i \sigma_{y} \\
\sigma_{x}-i \sigma_{y} & I_{2}-\sigma_{z}
\end{array}\right)
$$

we have $G_{1}=-1$ and $G_{2}=-3$, from which we get $a=-1, b=0$ and $c=-3$. According to the theorem, we obtain $P=0, Q=0$. Since $P^{3} / 27+Q^{2} / 4=0$ in this case, we have three real solutions $X_{1}=X_{2}=X_{3}=0$. Hence $c_{1}=c_{2}=c_{3}=\pi / 2$, and $t^{*}(S W A P)=\frac{1}{\pi J} \sum_{i=1}^{3} c_{i}=\frac{3}{2 J}$.

Therefore the Cartan decomposition of Swap is simply of the form

$$
S w a p=\exp \left(\frac{-\pi i}{4}\right) \exp \left(\frac{\pi i}{4}\left(\sigma_{x} \otimes \sigma_{x}+\sigma_{y} \otimes \sigma_{y}+\sigma_{z} \otimes \sigma_{z}\right)\right)
$$

From eq. (21) and the following formula

$$
\exp \left(\frac{i}{4} \sigma_{y} \otimes \sigma_{y}\right)=\left(\exp \left(-\frac{\pi i}{4} \sigma_{x}\right) \otimes \exp \left(\frac{\pi i}{4} \sigma_{x}\right)\right) \exp \left(-\frac{\pi}{4} i \sigma_{z} \otimes \sigma_{z}\right)\left(\exp \left(\frac{\pi i}{4} \sigma_{x}\right) \otimes \exp \left(-\frac{\pi i}{4} \sigma_{x}\right)\right)
$$

we have

$$
\begin{aligned}
\exp \left(\frac{\pi i}{4}\right) \text { Swap }= & \exp \left(-i \frac{1}{4}\left(H_{2}-H_{4}\right)\right) \exp \left(-i \frac{1}{2 J} H_{d}\right) . \\
& \exp \left(i \frac{1}{4}\left(H_{2}-H_{4}\right)\right) \exp \left(-i \frac{1}{4}\left(H_{1}-H_{3}\right)\right) . \\
& \exp \left(-i \frac{1}{2 J} H_{d}\right) \exp \left(-i\left(\frac{1}{4} H_{3}\right)\right) \exp \left(-i \frac{1}{2 J} H_{d}\right) \exp \left(i \frac{1}{4} H_{1}\right) .
\end{aligned}
$$

From the above expression, one can easily get the corresponding steps to implement Swap gate by choosing the control parameters in the control Hamiltonian.

Example 3. $\sqrt{S W A P}$ gate For this gate we have $G_{1}=i / 4, G_{2}=0$, which yields $a=0$, $b=1 / 4, c=0$ and $P=Q=0$. Similar to the Swap gate case, one has $P^{3} / 27+Q^{2} / 4=0$. Hence we get the solution, $c_{1}=c_{2}=c_{3}=\pi / 4$, and $t^{*}(\sqrt{S W A P})=\frac{3}{4 J}$. The gate can be implemented according to the following decomposition,

$$
\begin{aligned}
\exp \left(\frac{\pi i}{8}\right) \sqrt{S W A P}= & \exp \left(-i \frac{1}{4}\left(H_{2}-H_{4}\right)\right) \exp \left(-i \frac{1}{4 J} H_{d}\right) \cdot \\
& \exp \left(i \frac{1}{4}\left(H_{2}-H_{4}\right)\right) \exp \left(-i \frac{1}{4}\left(H_{1}-H_{3}\right)\right) . \\
& \exp \left(-i \frac{1}{4 J} H_{d}\right) \exp \left(-i\left(\frac{1}{4} H_{3}\right)\right) \exp \left(-i \frac{1}{4 J} H_{d}\right) \exp \left(i \frac{1}{4} H_{1}\right) .
\end{aligned}
$$

Example 4. Controlled- $U$ gate The controlled-U gate is of the form $C_{U}=|0\rangle\langle 0| \otimes I+$ $|1\rangle\langle 1| \otimes U$, where $U$ is an arbitrary single-qubit unitary operation, $U=\exp \left(i \gamma_{1} \sigma_{x}+i \gamma_{2} \sigma_{y}+i \gamma_{3} i \sigma_{z}\right)$, 
$\gamma_{1}, \gamma_{3}, \gamma_{3} \in \mathbb{R}$. The corresponding local invariants are $G_{1}=\cos ^{2} \gamma, G_{2}=2 \cos ^{2} \gamma+1$, where $\gamma=\sqrt{\gamma_{1}^{2}+\gamma_{2}^{2}+\gamma_{3}^{2}}$. Accordingly we have $a=\cos ^{2} \gamma, b=0$ and $c=2 \cos ^{2} \gamma+1$. As in this case one has $P=-\sin ^{4} \gamma / 3$ and $Q=-2 \sin ^{6} \gamma / 27$, the condition $P^{3} / 27+Q^{2} / 4=0$ is satisfied. Hence $X_{1}=2 \sin ^{2} \gamma / 3$ and $X_{2}=X_{3}=-\sin ^{2} \gamma 3$. Therefore $c_{1}=\arcsin \sqrt{X_{1}+(3-c) / 6}=\arcsin |\sin \gamma|$, $c_{2}=c_{3}=0$, and the minimal time to implement $C_{U}$ is $t^{*}\left(C_{U}\right)=\frac{1}{\pi J} \arcsin |\sin \gamma|$.

\section{Discussions}

By using the local invariants of unitary operators, we have presented an explicit formula of the minimal time required to implement a given unitary operator for the heteronuclear two-qubit quantum system. The formula can be easily used to compute the minimal time needed to implement the quantum gates such as $C_{N O T}$, SWAP and controlled-U ones. The protocols we presented for optimally implementing the quantum gates can be directly operated in the heteronuclear system [15]. Our idea, employing both the Cartan decomposition of a unitary operator and its local invariants, can be also used for computing the optimal control time for other quantum systems.

Acknowledgments This work is supported by the National Natural Science Foundation of China (Grant No. 11275131) and the National Research Foundation for the Doctoral Program of Higher Education of China.

\section{References}

[1] Warren W, Rabitz H, Dahleb M. Coherent control of quantum dynamics: the dream is alive. Science, 1993, 259: 1581-1589

[2] Rabitz H, de Vivie-Riedle R, Motzkus M, et al. Whither the future of controlling quantum phenomena? Science, 2000, 288: 824-828

[3] Daniel C, Full J, Gonzàlez L, et al. Deciphering the reaction dynamics underlying optimal control laser fields. Science, 2003, 299: 536-539

[4] Nielsen M A, Chuang I L. Quantum Computation and Quantum Information. Cambridge: Cambridge University Press, 2000 
[5] Ernst R R, Bodenhausen G, Wokaun A. Principles of Nuclear Magnetic Resonance in One and Two Dimensions. Oxford: Oxford University Press, 1987

[6] Gershenfeld N A, Chuang I L. Bulk spin-resonance quantum computation. Science, 1997, 275 : 350-356; Cory D G, Fahmy A, Havel T. Ensemble quantum computing by NMR spectroscopy. Proc Natl Acad Sci USA, 1997, 94: 1634-1639

[7] Lapert M, Salomon J, Sugny D. Time-optimal monotonically convergent algorithm with an application to the control of spin systems. Phys Rev A, 2012, 85: 033406

[8] Tibbetts K W M, Brif C, Grace M D, et al. Exploring the tradeoff between fidelity and time optimal control of quantum unitary transformations. Phys Rev A, 2012, 86: 062309

[9] Motzoi F, Gambetta J M, Merkel S T, et al. Optimal control methods for rapidly time-varying Hamiltonians. Phys Rev A, 2011, 84: 022307

[10] Assémat E, Lapert M, Zhang Y, et al. Simultaneous time-optimal control of the inversion of two spin-1/2 particles. Phys Rev A, 2010, 82: 013415

[11] Lapert M, Zhang Y, Braun M, et al. Singular extremals for the time-optimal control of dissipative spin 1/2 Particles. Phys Rev Lett, 2010, 104: 083001

[12] Khaneja N, Brockett R, Glaser S J. Time optimal control in spin systems. Phys Rev A, 2001, 63: 032308

[13] Makhlin Y. Nonlocal properties of two-qubit gates and mixed states, and the optimization of quantum computations. Quant Inf Proc, 2002, 1: 243-252

[14] Zhang J, Vala J, Sastry S, et al. Geometric theory of nonlocal two-qubit operations. Phys Rev A, 2003, 67: 042313

[15] Glaser J, Schulte-Herbrüggen T, Sieveking M, et al. Unitary control in quantum ensembles: maximizing signal intensity in coherent spectroscopy. Science, 1998, 280: 421-424 